\title{
La figure du père dans les romans de Jin Yong
}

Et la littérature populaire contemporaine chinoise

Figures of the Father in Jin Yong and Contemporary Chinese Folk Literature 金庸筆下的父親形象與中國當代通俗文學

\section{Nicolas Zufferey}

\section{CpenEdition \\ Journals}

Édition électronique

URL : http://journals.openedition.org/extremeorient/222

DOI : 10.4000/extremeorient.222

ISSN : 2108-7105

\section{Éditeur}

Presses universitaires de Vincennes

\section{Édition imprimée}

Date de publication : 1 janvier 2012

Pagination : 219-244

ISSN : 0754-5010

\section{Référence électronique}

Nicolas Zufferey, "La figure du père dans les romans de Jin Yong 》, Extrême-Orient Extrême-Occident [En ligne], Hors-série | 2012, mis en ligne le 01 janvier 2015, consulté le 21 avril 2019. URL : http:// journals.openedition.org/extremeorient/222 ; DOI : 10.4000/extremeorient.222 


\title{
La figure du père dans les romans de Jin Yong et la littérature populaire contemporaine chinoise
}

\author{
Nicolas Zufferey
}

Dans Millénium (2005-2007), la célèbre trilogie de Stieg Larsson, l'héroöne, Lisbeth Salander, tente à deux reprises de tuer son père: une première fois, alors qu'elle est encore enfant, en mettant le feu à sa voiture; et une deuxième fois, adulte, en lui arrachant une partie du visage avec une hache. Ce père est un monstre: entre autres crimes, il tire lui-même sur sa fille et la laisse pour morte; mais c'est tout de même son père. Pour en rester aux romans policiers scandinaves, les héros sont souvent de «mauvais pères »: toujours dans Millénium, le personnage principal, le journaliste Blomkvist, est divorcé et ne voit quasiment jamais sa fille; Wallender, le policier des romans de Henning Mankell, est lui aussi divorcé et entretient une relation difficile avec sa fille Linda; Erlendur, le commissaire des œuvres d'Arnaldur Indridason, a abandonné ses enfants alors qu'ils étaient encore en jeune âge, et se sent coupable des problèmes psychologiques qu'ils rencontrent en grandissant.

Ces figures de pères ratés reflètent jusqu'à un certain point les réalités sociales dans des sociétés modernes, avec le délitement de la famille et la remise en question des rôles sociaux «traditionnels ${ }^{1}{ }^{\circledR}$. Mais ces mauvais pères éprouvent une culpabilité très «occidentale»: on est frappé de voir à quel point cette littérature insiste sur les devoirs et les responsabilités des parents. À l'inverse, les enfants sont présentés comme de simples victimes, avec des droits qui peuvent paraître exorbitants, et notamment celui de critiquer leurs parents et d'invoquer les carences de ces derniers pour excuser leurs propres errements. On est très loin ici des sociétés traditionnelles - et de la culture chinoise ancienne, où les enfants ont surtout des devoirs.

1. La tradition et la modernité sont évidemment des représentations; elles sont en grande partie imaginées, voire inventées, et cela vaut encore plus dans des œuvres de fiction comme celles qui seront examinées dans cet article. 
La société chinoise ancienne est fortement patriarcale; le culte des ancêtres, la hiérarchisation du groupe, l'autorité du père, la piété filiale, l'obligation faite aux générations successives de produire une descendance masculine sont autant de traits typiques de ce modèle de société. Le père joue un rôle central, au sein de la famille bien sûr, mais aussi comme métaphore de l'autorité en général: l'empereur, les ministres, les chefs entretiennent avec leurs sujets et leurs subordonnés une relation qui se veut par bien des aspects analogue à celle qui régit les rapports entre un père et ses enfants.

Cette vision traditionnelle a laissé des traces dans la société chinoise moderne, mais avec des limites et des ambiguïtés typiques des sociétés en transition ou en voie de modernisation. La «littérature populaire ${ }^{2}$ » (tongsu wenxue) est un moyen privilégié pour appréhender ces évolutions ${ }^{3}$. Elle possède, comme son nom l'indique, une dimension populaire - c'est-à-dire qu'elle s'adresse à un public large, qui se reconnaît dans celle-ci, et dont elle reflète au moins en partie les valeurs. En un sens, elle reflète mieux la société profonde que la littérature «pure» (chun wenxue), issue de la «Nouvelle Littérature» des années 1910-1920, souvent plus élitaire, et parfois plus normative que descriptive; les «grands» auteurs, à commencer par Lu Xun, ont d'ailleurs reproché à la littérature populaire son manque d'engagement en faveur de la modernisation, voire son conservatisme ou sa nature «réactionnaire».

On ne peut nier une certaine tendance au conservatisme dans certaines œuvres populaires. À la fin des années 1910 et au début des années 1920, la piété filiale (xiao) est l'un des enjeux des débats entre «modernistes» et «conservateurs». Avocats de la «Nouvelle Culture» et littérateurs modernistes voient dans cette valeur «traditionnelle» un symbole de l'autoritarisme et des

2. Par «littérature populaire», on entend couramment des genres tels que le roman sentimental, le roman policier, le roman fantastique, etc., ou encore, en ce qui concerne le domaine chinois, le roman d'arts martiaux, par opposition à la «grande» littérature. À vrai dire, la littérature populaire échappe à la définition, et la notion même est largement rejetée par la critique. De façon empirique, on peut toutefois constater l'existence de genres dont les méthodes de production et de diffusion, ainsi que le public, diffèrent au moins partiellement de ceux de la «grande» littérature: une fiction de masse et de distraction, produite pour un lectorat large, généralement urbain, selon des méthodes commerciales et à des rythmes rapides, et vendue dans des endroits très ouverts (grandes surfaces, éventaires de marchés, kiosques à journaux, etc.).

3. Voir notamment Barthlein (1999). L'intérêt sociologique de la littérature populaire ne vaut bien entendu pas que pour la Chine, voir Loïc Artiaga à propos de la littérature populaire en France: «Par sa diversité générique, thématique et matérielle, le roman populaire joue pleinement pour l'explorateur du passé le rôle de révélateur des mutations culturelles, des usages de la fiction ou des fantasmes des époques qu'il a traversées. » Artiga (2008): 11. 
hiérarchies d'une Chine dépassée. À l'inverse, nombre d'œuvres populaires défendent la piété filiale, par exemple Père et fils (Fu zi, 1921), une nouvelle de Zhou Shoujuan (1865-1968) qui raconte le sacrifice d'un jeune homme particulièrement filial: ce fils a reçu une éducation moderne, et son père le maltraite, mais lorsque ce dernier tombe malade, le jeune homme n'hésite pas à donner son sang pour le sauver et meurt suite à la transfusion. Cette valorisation du sacrifice personnel pour le père, à une époque où la littérature engagée prône l'émancipation de la jeunesse, valut bien des critiques à Zhou Shoujuan de la part de «modernistes » comme Zheng Zhenduo (1898-1958) ou Guo Moruo (1892-1978) ${ }^{4}$. Zhou Shoujuan était pourtant loin d'être le plus rétrograde des auteurs de l'époque, et d'autres auteurs populaires allèrent beaucoup plus loin que lui dans le refus des nouvelles idées, qui selon eux mettaient en danger la morale chinoise. Wang Dungen (1888-?), un auteur généralement rapproché de la littérature sentimentale «des canards mandarins et des papillons » (yuanyang hudie pai), décrit par exemple dans l'une de ses œuvres l'ingratitude d'enfants qui n'éprouvent aucune reconnaissance envers leurs parents et justifient cette attitude en invoquant des théories selon lesquelles les enfants ne doivent rien à des géniteurs qui ne les ont conçus que pour assouvir leurs instincts sexuels ${ }^{5}$. Cette vision purement «biologique» des rapports parents/enfants, qui remet en cause d'une manière caricaturale le devoir de piété filiale, est dénoncée comme immorale et présentée comme importée de l'Occident ${ }^{6}$. Certains romanciers conservateurs vont jusqu'à traiter de «bandits» (qiangdao) les détracteurs des valeurs traditionnelles, voyant dans le manque de piété filiale la faute la plus grave $^{7}$. Au début des années 1920, puis dans les années 1930, la littérature populaire représente donc une sorte de bastion pour les valeurs conservatrices; elle touche un large public et fait l'objet d'adaptations au cinéma, un cinéma très populaire qui, au début des années 1930, tend aussi au conservatisme ${ }^{8}$. On comprend donc les attaques parfois virulentes des tenants de la «Nouvelle Culture», ces auteurs qui seront par la suite placés sur l'autel de l'orthodoxie par les historiens de la littérature: Lu Xun, Mao Dun ou encore Ba Jin (Pa Kin), dont le roman Famille (1933) représente l'une des condamnations les plus féroces du système patriarcal traditionnel.

4. $\quad C f$. Tang Zhesheng (2008): 32-33

5. Cf. ibid.: 33-34.

6. Cf. ibid.: 34. Cette interprétation très matérialiste de la procréation des enfants n'est certainement pas l'apanage de l'Occident, on la trouve déjà en Chine ancienne, par exemple dans le Lunheng (Discussions critiques) de Wang Chong (27-97?), chap. 14, «Wu shi».

7. Cf. ibid.: 33 .

8. Sur le conservatisme du cinéma des années 1930, cf. Pickowicz (1991). 
Si certains reproches à l'endroit de la littérature populaire sont compréhensibles, l'idée sous-jacente d'une opposition radicale entre une littérature populaire qui ne serait que «conservatrice» et une «grande» littérature progressiste n'est pas convaincante: la littérature populaire reflète aussi bien la «tradition» que la «modernité»; elle représente elle aussi un pan important de la littérature chinoise moderne ${ }^{9}$. En ce qui concerne les valeurs patriarcales, ou «traditionnelles », bien des œuvres décrivent simplement la nouvelle société telle qu'elle est, avec à la fois la survivance de traits traditionnels et l'influence de conceptions modernes, sans forcément porter de jugement sur les uns ou les autres.

Dans cet article, je m'intéresserai à la figure du père dans un genre populaire qui ne se donne certainement pas pour priorité de refléter les réalités sociales, à savoir le «roman d'arts martiaux» (wuxia xiaoshuo). Il sera intéressant de voir comment un genre apparemment très éloigné de la Chine actuelle peut cependant être révélateur des évolutions sociales récentes. Étant donné l'immense richesse de la littérature populaire en Chine, ce travail ne prétend pas proposer un tableau représentatif de celle-ci, mais les œuvres examinées donneront tout de même une bonne idée du statut du père et des valeurs patriarcales dans un contexte de crise morale, d'hybridation culturelle et de transformation en profondeur de la société chinoise.

La littérature d'arts martiaux possède des origines anciennes, mais elle existe en tant que genre à part entière à partir de la deuxième moitié du XIX ${ }^{\mathrm{e}}$ siècle. La Shanghai des années 1920-1930 est un âge d'or pour les genres populaires en général, et pour le roman d'arts martiaux en particulier. Après 1949, la Chine continentale connaît trois décennies pauvres en l'espèce, mais la littérature populaire survit aux marges de l'Empire, principalement à Hong Kong et à Taiwan : à Hong Kong, dans les années 1950, la littérature d'arts martiaux connaît un renouveau (connu sous le nom de «Nouveau roman d'arts martiaux», Xin wuxia xiaoshuo). Dans les années 1980, la littérature populaire se réimplante en Chine continentale, dans un premier temps comme forme importée de Hong Kong et de Taiwan. Depuis les années 1990, la production est foisonnante, dans les genres habituels de la littérature populaire, parfois très occidentalisés, comme le roman policier, le roman fantastique, le roman de science-fiction, mais aussi sous des

9. Les premières œuvres de la littérature chinoise «moderne» sont d'ailleurs des romans populaires. Le critique Tang Zhesheng fait du roman de Han Bangqing, Haishang hua liezhuan, publié en 1892, la première œuvre de la littérature moderne (Cf. Tang Zhesheng, Zhongguo xiandai tongsu xiaoshuo sibianlu: 1-2), soit plusieurs décennies avec les premiers romans et nouvelles de la «Nouvelle Littérature» (Xin wenxue). La littérature moderne ne se réduit donc pas à la «Nouvelle Littérature», même si celle-ci, principalement pour des raisons politiques, s'est imposée comme orthodoxie dans les manuels d'histoire de la littérature. 
formes beaucoup plus «chinoises», au premier rang desquelles le roman d'arts martiaux et le roman historique.

Je me concentrerai sur la figure du père dans l'œuvre de Jin Yong (Louis Cha, 1924-), le plus populaire des auteurs de romans d'arts martiaux du xx ${ }^{\mathrm{e}}$ siècle, qui signa entre le milieu des années 1950 et le début des années 1970 une quinzaine d'œuvres, d'abord proposées au public sous la forme de feuilletons dans des journaux, avant d'être publiées comme livres. Les œuvres de Jin Yong connurent un succès immense, et encore aujourd'hui, le nom de cet auteur est connu de tous les Chinois. Cette popularité s'explique par toutes sortes de raisons, la première étant sans doute la qualité de l'écriture de Jin Yong: Jin Yong est un styliste accompli et un superbe conteur, à l'imagination débordante, et son œuvre est traversée d'une vie et d'un humour qui tranchent avec l'ambiance souvent morose de la littérature chinoise du $\mathrm{xx}^{\mathrm{e}}$ siècle. Mais ce succès s'explique sans doute aussi par le fait que les lecteurs se reconnaissent dans un genre qui s'inscrit dans la tradition de la culture chinoise ancienne, celle du roman Les Bords de l'eau, par exemple.

À proprement parler, Jin Yong est un auteur hongkongais, et de fait, il n'aurait pas pu publier son œuvre en Chine populaire étant donné le contexte politique de l'époque. Sous une forme traditionnelle, les romans de Jin Yong manifestent des aspects résolument modernes; la construction du récit, l'humour et d'autres éléments narratifs sont à certains égards proches du roman occidental - Jin Yong lui-même avoue quelques filiations européennes. On peut aussi expliquer certaines caractéristiques de ses œuvres comme reflétant l'esprit d'une grande métropole capitaliste tournée vers le commerce. L'idéal politique de Jin Yong semble relativement «libéral» (au sens français du terme). Notre auteur se plaît par ailleurs à mettre en scène des personnages hybrides (mi-chinois, miétrangers) ${ }^{10}$, trait qu'on sera tenté de mettre en lien avec le cosmopolitisme de Hong Kong. Nous verrons par ailleurs que le père chez Jin Yong est souvent absent ou indigne, aspect que l'on peut sans doute interpréter comme reflétant l'identité particulière d'une colonie arrachée depuis plus d'un siècle à la mèrepatrie. En d'autres termes, la «patrie» de Jin Yong est pour le moins ambiguë, et cela n'est pas inintéressant dans la perspective d'une réflexion sur le père.

En réalité, dans l'œuvre de Jin Yong, le père est à la fois absent et omniprésent. La plupart des héros n'ont plus leurs parents, qu'ils soient orphelins

10. Plusieurs des personnages importants de Jin Yong sont de sang mélangé, à la fois Han et «barbare» : un cas exemplaire est Qiao Feng (Xiao Feng), le héros de Demi-dieux et semi-dragons (Tianlong babu), qui se découvre une ascendance Khitan; Jin Yong affuble par ailleurs l'empereur mandchou Qianlong d'ancêtres chinois dans Le Livre et l'Épée (Shu jian enchou lu). Plusieurs personnages «trahissent» leur « race», par exemple la princesse mongole Zhao Min dans Le Céleste Tueur de dragons (Yitian tulongji). 
depuis leur tendre enfance ou qu'ils les aient perdus plus tard, souvent dans des circonstances violentes. Les géniteurs sont donc physiquement absents, mais en même temps ils sont présents en creux, puisque la disparition des parents pèse sur la destinée et les actes de leur progéniture : la quête de l'identité personnelle ou des origines familiales, la recherche d'un père ou d'une mère perdus, ou le devoir de vengeance (la vengeance des parents morts est l'une des constantes du genre) sont en effet quelques-uns des principaux moteurs de l'action.

La figure du père est par ailleurs présente sous la forme du maître d'arts martiaux, qui remplit pour ses disciples, d'ailleurs organisés en fratries (selon l'ancienneté), la fonction de père. Comme le dit le critique Zhang Yinde, «en l'absence d'un père biologique, le maître en arts martiaux remplit le rôle paternel en transmettant au disciple-fils les secrets des armes ${ }^{11} »$. Les disciples s'adressent à leur maître en l'appelant shifu, soit littéralement «père-maître». La relation maître-disciple est fréquemment mise sur le même plan que la relation père-fils, comme le rappelle le narrateur parfois pontifiant du roman Les Compagnons aux aigles merveilleux:

La dynastie Song attachait beaucoup d'importance aux rites, et les relations hiérarchiques entre maître et disciple étaient mises sur le même plan que les relations entre un prince et ses sujets, ou entre un père et ses fils - il n'était pas question d'enfreindre ces hiérarchies. (Shendiao xialü, chap. $14^{12}$ )

Un exemple typique, parmi d'autres, est le personnage principal de Aventurier fier et souriant, Linghu Chong, un orphelin qui considère explicitement son maître, Yue Buqun, comme son véritable père. La présence du père dans les romans de Jin Yong est donc un peu paradoxale, entre des géniteurs qui pèsent lourdement par leur absence, et des maîtres qui reprennent une partie du rôle et des attributs des pères.

Les enfants ont de multiples devoirs envers leur père ou leur maître, nous le verrons, mais ces devoirs sont en principe fondés sur l'attachement naturel

11. Zhang Yinde (2003): 331.

12. Étant donné le grand nombre d'éditions, légales ou pirates, imprimées ou sur Internet, des œuvres de Jin Yong, je renonce à indiquer une pagination renvoyant à une édition précise - nous indiquons simplement le numéro du chapitre. Les titres des romans de Jin Yong sont souvent difficiles à traduire; nos traductions de ces titres sont parfois inspirées de listes qu'on trouve sur Internet, $c f$. par exemple http://fr.wikipedia.org/wiki/Jin_Yong (consulté le 14 février 2011). Un seul roman de Jin Yong est traduit en français, $\mathrm{La}$ Légende du héros chasseur d'aigles (Shediao yingxiong zhuan); malheureusement, le deuxième tome de cette traduction est un condensé bâclé des tomes 2 à 4 de l'original. En anglais, la situation est meilleure, on recommandera particulièrement la traduction de Le Cerf et le Tripode (Lu ding ji): John Milford et Rachel May, The Deer and the Cauldron (1997-2003). 
qu'on éprouve vis-à-vis des parents. Jin Yong s'inscrit ici implicitement dans la tradition de Confucius, qui manifeste à plusieurs reprises l'importance des sentiments dans la piété filiale, le deuil ou les rites; Confucius est par exemple effondré lorsque meurt son disciple Yan Yuan, qu'il considère presque comme son fils ${ }^{13}$. Chez Jin Yong, parents et enfants sont souvent liés par des sentiments très forts. Dans Le Héros chasseur d'aigles, Huang l'Herboriste, qui pourtant est loin d'être un tendre, éprouve pour sa fille Huang Rong une affection inconditionnelle:

Huang Rong était la fille unique et adorée de Huang l'Herboriste. Au moment de la naissance de Huang Rong, sa mère avait été confrontée à des problèmes qui l'avaient épuisée, avec pour conséquence des complications et son décès lors de l'accouchement. Comme Huang l'Herboriste avait chassé tous ses disciples de l'île, il n'y restait plus que le père et la fille pour se soutenir mutuellement. Huang l'Herboriste, surnommé «L'Hérétique de l'Est», avait un comportement excentrique, répétant que les règles et rites du monde ne valaient pas mieux que des pets de chien; il ne chercha donc nullement à mettre la bride à ses sentiments pour sa fille, qu'il aimait plus que sa vie, la gâtant tant et si bien qu'elle devint capricieuse et indisciplinée. (Shediao yingxiong zhuan, chap. 10)

La mort des parents est évidemment tragique - dans Aventurier fier et souriant, la détresse du jeune Lin Pingzhi lorsqu'il trouve ses parents morts est particulièrement touchante. Il n'y a rien là que de très banal. Mais dans certains cas, la force des sentiments pour les parents peut paraître plus difficile à comprendre, et s'explique sans doute aussi par un contexte socioculturel dans lequel le père représente un repère incontournable. Le héros de Biographie non officielle de Renard, Hu Fei, n'a jamais connu ses parents, mais il est effondré lorsqu'il apprend qu'ils ont été assassinés : il se jette au sol en sanglotant et jure de les venger (Fei hu wai zhuan, chap. 11). Les sentiments pour les parents impliquent donc une dimension culturelle, ils ne sont pas forcément personnalisés : si on pleure ses parents ou ses enfants, c'est aussi parce qu'être privé des uns ou des autres est tout simplement anormal et difficile à vivre dans une société patriarcale. Cette importance apparaît fort bien dans le passage suivant, tiré de Demi-dieux et semi-dragons, où l'assassin Duan Yanqing se réjouit d'apprendre que Duan Yu, l'un des héros du roman, est en réalité son fils:

Duan Yanqing, qui n'avait jamais connu le bonheur d'avoir des enfants et une famille, apprenait soudain que dans ce monde il y avait un enfant qui était son propre fils! C'était un bonheur indescriptible, et il avait d'un coup l'impression que la réputation

13. Sur l'affection entre parents et enfants, ou maîtres et disciples, chez Confucius, voir Entretiens de Confucius (Lunyu), chap. 4.21, 17.21, 19.14; voir aussi 11.10 et 11.11. 
ou la gloire, les titres royaux ou un fief, ne valaient de loin pas l'honneur d'avoir un fils ! Joie et étonnement se bousculaient en lui, il avait envie de sauter en hurlant d'allégresse - et avec un grand «Klung ! » le bâton de fer qu'il tenait à la main tomba au sol. (Tianlong babu, chap. 48)

Les sentiments ne se distinguent pas non plus radicalement des devoirs, comme en témoigne un passage où Duan Yu, en voyage au royaume des Xixia, est interrogé sur «les gens qu'il préfère au monde». Le jeune homme est tenté de dire que c'est sa bien-aimée, Wang Yuyan, qu'il chérit le plus, mais il se ravise:

Il songea soudain qu'il était prince-héritier du Roi Pacificateur-du-Sud de Dali, que chez les Xixia, chacune de ses paroles aurait des répercussions sur l'image de son pays; que s'il perdait la face, ce n'était pas grave, mais qu'il ne pouvait nuire à la réputation de son royaume de Dali. Il dit alors: "C'est bien entendu mon père et ma mère que je préfère. » En prononçant les deux mots «père» et «mère», il sentit naturellement ce que signifiait aimer ses parents, et il se dit que l'amour pour ses parents et l'amour pour Wang Yuyan étaient deux choses tout à fait différentes - il était difficile de dire lequel était le plus profond, mais il ne mentait certainement nullement en affirmant comme il venait de le faire que c'était son père et sa mère qu'il préférait au monde. (Tianlong babu, chap. 46)

À vrai dire, dans la morale traditionnelle comme dans l'œuvre de Jin Yong, les sentiments comptent moins que les devoirs. Ne pas aimer ses parents, voire les détester, ne libère pas des obligations à leur égard. Un cas frappant est celui de Yuan Ziyi, l'une des héroïnes les plus mélancoliques de Jin Yong. Dans Biographie non officielle de Renard, elle explique à Hu Fei à quel point elle hait son père Feng Tiannan, qui a violé sa mère (elle-même est le fruit de ce viol):

Ce pervers de Feng Tiannan mérite plus que la mort. Que ne puis-je le poignarder de ma propre main! [...] Ma haine est cent fois plus forte que celle que tu éprouves pour lui ! Tu ne le hais que depuis quelques mois, moi je l'ai haï toute ma vie. (Feihu waizhuan, chap. 14)

Cette haine, cependant, n'empêche pas la jeune fille de sauver son père à trois reprises, obéissant en cela aux injonctions de son maître:

Ton père a commis une multitude de crimes, il est voué à être frappé par le malheur. Mais tu dois lui sauver trois fois la vie afin de remplir les devoirs qu'une fille doit à son père. Une fois cela fait, tu n'auras plus rien à faire avec lui. (ibid.)

De la même façon, Duan Yu veut tuer Duan Yanqing, dont il vient d'apprendre qu'il est son père, mais qui n'en reste pas moins l'ennemi mortel de la famille. Sa mère l'en dissuade: 
Tu commettrais un parricide ! Je n'ai jamais aimé cet homme, mais... mais il ne faut pas que cela te fasse commettre un crime, avec pour conséquence funeste qu'à ta mort tu te retrouverais dans l'enfer d'Avici et serais privé du paradis de l'Ouest... Je ne voulais pas te dire [que Duan Yanqing est ton vrai père], mais maintenant je n'ai pas le choix, je suis obligée de te le dire... (Tianlong babu, chap. 48)

Pourquoi sauver ou épargner des géniteurs aussi indignes? Pour remplir ce devoir de réciprocité (bao), fondamental dans la morale traditionnelle, selon lequel on doit rendre à ses parents ce qu'ils nous ont donné, à commencer par la vie: comme le rappelle Yuan Ziyi elle-même, «sans père et mère, comment serait-on là?» (Feihu waizhuan, chap. 14). Les parents nous ont mis au monde, et il est tout simplement naturel de leur rendre ce «don», même si, comme dans le cas de Yuan Ziyi ou de Duan Yu, les géniteurs n'ont certainement pas conçu leurs enfants par amour et ne s'en sont jamais occupés. Il s'agit d'idées qu'on peut rapprocher du confucianisme : les «trois vies » que Yuan Ziyi accorde à son père rappellent le deuil de trois ans qu'on doit garder à la mort de ses parents, période qui elle-même correspond aux trois ans de la petite enfance où l'on dépend de leur soutien pour sa survie ${ }^{14}$. Les devoirs de Yuan Ziyi et de Duan Yu envers leurs géniteurs rappellent également la piété filiale de sages mythiques de la tradition chinoise, par exemple celle du roi Shun, qui continua à servir avec dévotion un père qui pourtant le traitait d'une manière très injuste.

Dans les romans de Jin Yong, un autre devoir important vis-à-vis du père ou du maître est l'obéissance, ce qui à nouveau correspond à l'une des premières exigences du confucianisme ${ }^{15}$. La famille comme les écoles d'arts martiaux sont organisées d'une façon très hiérarchique, et la parole du père ou du maître ne souffre aucune discussion. Dans Les Compagnons aux aigles merveilleux, le narrateur rappelle la force de cette autorité:

À cette époque, les statuts respectifs du prince par rapport aux sujets, du père par rapport aux fils, du maître par rapport aux disciples, étaient très stricts - lorsque le prince ordonnait à un sujet de mourir, celui-ci n'osait pas ne pas mourir; lorsque le père ordonnait à son fils de mourir, celui-ci ne pouvait désobéir; et de la même façon dans les relations hiérarchiques entre maîtres et disciples, il n'y avait aucune place pour la moindre incartade. (Shendiao xialï, chap. 11)

L'obéissance est un devoir même lorsque les maîtres paraissent excessivement sévères. Dans Aventurier fier et souriant, Linghu Chong, qui pourtant est loin d'être un personnage soumis, obéit sans broncher lorsque son maître Yue Buqun le punit d'un an d'isolement complet au sommet d'une montagne. L'autorité du

14. Cf. ibid.: 17.21 .

15. Cf. ibid.: 2.5 . 
père ou du maître paraît particulièrement intimidante. Dans Le Héros chasseur d'aigles, lorsqu' on dit à Mei Chaofeng, elle-même une femme redoutable, que son maître Huang l'Herboriste est venu pour la punir, elle est épouvantée:

Elle n'eut qu'un seul désir, celui de détaler, mais malheureusement comment l'auraitelle pu avec ses jambes paralysées? Elle était si épouvantée que ses âmes menaçaient de s'envoler, que ses dents s'entrechoquaient... elle se sentait complètement perdue [...]. Elle pensa : «Mon Maître, ici ? Comment me mettra-t-il à mort ?» Songeant à la cruauté naturelle de Huang l'Herboriste, à ses méthodes terribles, elle blêmit et fut prise de tremblements. Elle se représenta son maître, avec son visage impitoyable, se tenant debout devant elle, et elle se sentit soudain très faible, comme si elle avait oublié toutes ses techniques de combat. Elle lui dirait, prosternée et tremblante: «Votre disciple mérite mille fois la mort, puissiez-vous seulement prendre en pitié le fait que je suis aveugle et à moitié paralysée, et me consentir une mort rapide ! Votre disciple s'est comportée d'une manière indigne et ne vaut pas mieux qu'un chien ou un cochon! » Puis, songeant à l'ancienne bienveillance de Huang l'Herboriste à son égard, sa crainte se transforma d'un coup en honte, et elle dit: «Mais non, Maître, vous n'avez pas à m'accorder une mort rapide! Plus votre châtiment sera sévère, mieux cela vaudra!» (Shediao yingxiong zhuan, chap. 10)

Mei Chaofeng a trahi son maître, sa faute est donc particulièrement lourde. La loyauté, en effet, est un autre devoir absolu vis-à-vis du père ou du maître. Là encore, les romans de Jin Yong s'accordent avec la morale confucianiste ${ }^{16}$. Trahir son père ou son maître est un crime - c'est une faute beaucoup plus grave, dans les romans d'arts martiaux, que le meurtre d'innocents, voire de frères ou de condisciples. La hiérarchie des crimes est évidente dans le passage suivant de $L a$ Biographie non officielle de Renard, où une jeune héroïne accuse ses condisciples :

Frère Murong, sœur Xue, je n'avais pas l'intention de vous faire du mal. Mais vous avez trahi notre école d'arts martiaux, et pris pour maître l'ennemi de notre maître ; en plus vous avez tué le deuxième disciple et son fils. Il y a là de quoi scandaliser à la fois le Ciel et les hommes! Si je ne punis pas cette trahison à l'égard de notre maître, sa réputation ne sera-t-elle pas piétinée par nos ennemis et leurs disciples? Et si je ne punis pas votre meurtre, les deux défunts n'erreront-ils pas à jamais dans les enfers avec un sentiment d'injustice? (Feihu waizhuan, chap. 20)

La loyauté implique bien entendu le sacrifice. Lorsque Duan Yu a été capturé à la place de celui qu'il croit encore être son père, il s'exclame:

Si je subis le grand malheur (de mourir) à la place de mon père, il n'y aura pas de quoi me plaindre. Ce sera même inespéré. (Tianlong babu, chap. 47)

16. Cf. ibid.: 13.18 
Dans Aventurier fier et souriant, l'obéissance et la loyauté prennent une forme caricaturale, avec des disciples qui témoignent à l'égard de leurs maîtres d'une dévotion malsaine. Dans l'extrait suivant, des partisans acclament leur maître Ren Woxing:

La clameur ébranlait ciel et terre, et les braves qui se tenaient debout au pied du pic se mirent à répéter à l'unisson: "Que notre maître sacré connaisse mille automnes et dix mille ans, qu'il unifie le monde des rivières et des lacs!» Les montagnes aux quatre directions renvoyaient ces cris en écho. Ren Woxing, radieux, se leva. À cette vue, tous ses disciples tombèrent à genoux, comme un seul homme, se prosternant sur le sol. D'un coup, le silence enveloppa le Pic de la Lumière Matinale. On n'entendait plus le moindre bruit. Le soleil levant inonda le visage, puis le corps, de Ren Woxing. Le grand maître de la Secte sacrée du Soleil et de la Lune imposait à tous son terrifiant prestige, tel une divinité céleste. (Xiao'ao jianghu, chap. 39)

Aventurier fier et souriant, publié à Hong Kong en 1967, a souvent été interprété comme une critique de la Révolution culturelle qui sévissait alors en Chine: Jin Yong, en mettant en scène des maîtres tout-puissants et des zélateurs fidèles jusqu'à la veulerie, aurait voulu critiquer le culte du président Mao. Ren Woxing dans le soleil levant, c'est le Grand Timonier dans la lumière rayonnante des affiches de propagande de la Révolution culturelle. Suggérer le président Mao sous les traits d'un «père-maître» (shifu) n'est pas sans pertinence : le fondateur de la république populaire de Chine joue dans l'inconscient des adolescents qui le vénèrent le rôle du père, un père qui parfois remplace le père biologique trop faillible, et qu'on doit parfois même dénoncer aux autorités. La Révolution culturelle pousse à la soumission inconditionnelle au père de la Nouvelle Chine, tout en tolérant, voire encourageant, la condamnation du géniteur. De ce point de vue, cette période tranche avec l'idée courante dans la tradition chinoise selon laquelle la loyauté envers le chef est un prolongement de l'obéissance envers le père. Dans la représentation qu'en propose Jin Yong, cependant, la Révolution culturelle reprend la vision confucianiste qui fonde l'autorité politique dans la loyauté due au père, dans une métaphore qui confère au chef un prestige et un pouvoir immenses.

La fidélité au père ou au maître implique aussi le devoir de vengeance, qui joue un rôle central dans les romans d'arts martiaux, où il représente l'un des principaux moteurs de l'action. Là aussi, les personnages s'inscrivent dans une tradition «confucianiste», et les textes classiques qui justifient, voire encouragent la vengeance de sang, sont fréquemment invoqués : ainsi le héros de La Biographie non officielle de Renard reprend-il l'énoncé du Classique des Rites (Liji, «Tangong, shang») selon lequel « on ne peut vivre sous le même ciel que 
l'ennemi de son père» (Feihu waizhuan, chap. 11) ${ }^{17}$. La vengeance obéit donc à une double logique, narrative et morale, dans les romans de Jin Yong, et les personnages de vengeurs y abondent: parmi les figures centrales mues par le désir de vengeance, mentionnons Yang Guo dans Les Compagnons aux aigles merveilleux, Hu Fei dans La Biographie non officielle de Renard, ou encore Lin Pingzhi dans Aventurier fier et souriant; mais d'une façon plus ponctuelle, presque tous les héros de Jin Yong sont confrontés, à un moment ou à un autre, au devoir ou au désir de vengeance.

La place centrale du père dans la tradition chinoise a des conséquences sur le statut de la femme; la société patriarcale présuppose par principe une séparation nette des sexes, et cela vaut pour la Chine ancienne, avec une hiérarchie très claire entre les genres, et des rôles et devoirs nettement distincts pour les hommes et les femmes. Selon la formule du Classique des rites, «les hommes ne parlent pas des affaires intérieures, les femmes ne parlent pas des affaires extérieures» $(\mathrm{Li} J i$, «Nei ze»). Cette «homosocialité» se retrouve dans les romans d'arts martiaux ${ }^{18}$.

Certes, on peut avoir l'impression d'un certain féminisme chez Jin Yong, qui met volontiers en scène des héroïnes très libres, et parfois beaucoup plus intelligentes que les protagonistes masculins. Mais que ce soit par fidélité aux codes du genre ou parce que Jin Yong lui-même est imprégné de valeurs traditionnelles, son œuvre ne remet généralement pas en question les valeurs patriarcales dominantes. Les personnages de jeunes filles fortes et astucieuses ne sont pas une invention de Jin Yong: ils abondent dans la littérature chinoise ancienne ${ }^{19}$. Mais que ce soit chez Jin Yong ou chez ses précurseurs, ces filles libres n'en doivent pas moins entrer dans le rang à un certain moment, c'est-à-dire se marier ${ }^{20}$. On retrouve ici un trait courant dans bien des sociétés traditionnelles, à savoir l'opposition entre l'âge de l'enfance, où la fille vit relativement librement, et

17. Cf. aussi Tianlong babu, chap. 48, pour une référence analogue.

18. L'homosocialité, à distinguer de l'homosexualité (même si, dans certains cas, les deux peuvent se recouper), privilégie les rapports sociaux entre personnes du même sexe - et donc la séparation plus ou moins radicale des sexes.

19. Sur les femmes d'épée et autres justicières (nüxia) dans la littérature chinoise ancienne, $c f$. Roland Altenburger (2009).

20. Un exemple de normalisation de ce type est Shisanmei, l'héroïne du roman Ernü yingxiong zhuan, publié dans le troisième quart du XIX $\mathrm{X}^{\mathrm{e}}$ siècle. Shisanmei est une justicière libre et sans scrupules, mais qui se débarrasse de son épée lorsqu'elle se marie. Cf. Roland Altenburger (2009): chap. 7, 227-286. 
l'âge nubile, où la femme se voit confinée à l'intérieur de la maison, sous la surveillance de son époux ${ }^{21}$.

L'un des personnages les plus révélateurs des limites du féminisme de Jin Yong est Huang Rong, qui apparaît dans deux romans, Le Héros chasseur d'aigles et Les Compagnons aux aigles merveilleux. Dans le premier de ces romans, Huang Rong est une jeune fille libre et insouciante, forte et parfois même cruelle, qui ne craint personne et se moque des conventions. Elle est amoureuse de Guo Jing, le héros, mais le maître de celui-ci, un moine taoïste, voudrait lui faire épouser une autre prétendante. Furieuse, Huang Rong imagine forcer le vieux moine à épouser une méchante grosse femme qu'ils viennent de rencontrer sur la route. Comme Guo Jing lui fait remarquer que le moine n'en voudrait pas, elle lui dit:

Bien sûr qu'il n'en voudrait pas! Mais il n'a qu'à réfléchir un peu : tu dis que tu ne veux pas épouser la demoiselle $\mathrm{Mu}$, alors pourquoi te force-t-il à le faire? Un jour, quand je serai plus forte que ce vieux taoïste, je le contraindrai à épouser une femme méchante et laide, pour qu'il goûte un peu au plaisir du mariage forcé. (Shediao yingxiong zhuan, chap. 11, trad. La Légende du héros chasseur d'aigles, vol. I: 545)

Dans ce passage, Huang Rong paraît donc remettre en cause l'un des piliers du système patriarcal, à savoir le mariage arrangé par les parents ou l'autorité. Mais dans Les Compagnons aux aigles merveilleux, où elle apparaît avec quelques années de plus, mariée et mère, elle joue un rôle très différent: elle se montre beaucoup plus conservatrice, comme nous le verrons ci-dessous ${ }^{22}$. Avec le mariage, elle a retrouvé la place normale de la femme dans la société, c'est-à-dire au sein de la famille, sous l'autorité du mari, et comme gardienne des valeurs patriarcales.

Pour en finir avec le féminisme de Jin Yong, signalons que notre auteur paraît à l'occasion faire preuve de beaucoup de tolérance vis-à-vis de personnages à la moralité plus que douteuse dans leurs relations avec les femmes; un bon exemple est Tian Boguang, dans Aventurier fier et souriant, un violeur éhonté que l'auteur parvient à nous présenter sous un jour plutôt sympathique... Dans La Biographie non officielle de Renard, le viol d'une marchande de poisson est rapporté d'une manière pour le moins désinvolte:

21. Cela vaut également pour l'Occident: le cinéma hollywoodien ou européen du milieu $\mathrm{du} \mathrm{Xx}^{\mathrm{e}}$ siècle propose fréquemment des processus de normalisation analogues, avec des jeunes filles rebelles qui se rangent une fois mariées.

22. Pour une appréciation du féminisme de Jin Yong, voir Tang Zhesheng (2007) : 178-180. 
Ce Feng disposait chez lui de femmes et de concubines, mais cela ne lui suffisait pas, et il prit [Sœur $\mathrm{Gu}$ ] de force et la souilla. Déboussolée, oubliant de se faire payer son poisson, elle s'enfuit chez elle. (Feihu waizhuan, chap. 14)

Dans cette brève description, le viol est en quelque sorte mis sur le même plan qu'un poisson non payé.

L'œuvre de Jin Yong met donc en scène un univers qui pour l'essentiel demeure homosocial, voire machiste ${ }^{23}$. La modernité, cependant, s'invite dans certains de ses romans, qui proposent parfois un message beaucoup moins conservateur. C'est le cas de deux romans en particulier, Les Compagnons aux aigles merveilleux et Le Cerf et le Tripode, qui vont assez loin dans la déconstruction du modèle patriarcal. Nous n'insisterons pas sur Le Cerf et le Tripode (Lu ding ji, 1970), parfois considéré comme le meilleur roman de Jin Yong, mais qui n'est guère représentatif de son œuvre, puisqu'il ne s'agit pas à proprement parler d'un roman d'arts martiaux. Disons simplement que Wei Xiaobao, le héros (ou l'antihéros), se trouve à tous égards aux antipodes de la morale traditionnelle : bâtard sans père, fils d'une prostituée, il a grandi dans un bordel; il est paresseux, menteur et peu scrupuleux dans le choix des moyens pour arriver à ses fins ; jouisseur invétéré, il manifeste la plus grande désinvolture vis-à-vis du mariage. En un mot, Wei Xiaobao est un personnage sympathique à de multiples égards, mais qui n'a rien d'un fils ou d'un père respectable.

Nous nous attarderons un peu plus sur Les Compagnons aux aigles merveilleux, l'un des romans les plus «psychologiques» de Jin Yong, qui a l'intérêt de poser d'une manière très directe la question du mariage et de la hiérarchie entre les générations. Le héros, Yang Guo, se comporte généralement d'une façon beaucoup plus morale que le héros de Le Cerf et le Tripode, mais il pèche contre la vertu confucianiste sur un point essentiel: il se montre insoumis et ne respecte pas les hiérarchies. Son prénom, «Guo» (littéralement, «passer», « dépasser»), est révélateur: si dans le roman ce prénom est expliqué dans un sens moral, comme signifiant «s'amender», « corriger ses fautes » (Shendiao xialü, chap. 14), on est

23. La littérature d'arts martiaux peut parfois se montrer très peu féministe; le célèbre auteur taïwanais Gu Long (1938-1985) fut souvent critiqué pour des jugements à l'emporte-pièce sur les femmes, et pour avoir mis en scène des personnages féminins particulièrement caricaturaux, qui ne semblent être là que pour nourrir les fantasmes des hommes. $C f$. Tang Zhesheng (2007): 193-194. Dans l'un des romans les plus célèbres de Gu Long, Duo qing jianke wuqing jian, publié en 1968, le héros Li Xunhuan émet une multitude d'opinions simplistes et cyniques sur le danger que les femmes représentent pour les hommes et sur le fait que ces derniers sont en définitive incapables de les comprendre: «Dans ce monde aucun homme ne peut vraiment comprendre une femme - celui qui croirait vraiment comprendre une femme souffrirait encore plus que les autres.» (chap. 40) 
tenté de l'interpréter comme signifiant au contraire «celui qui faute», « celui qui transgresse », tant le jeune homme respecte peu les normes. Yang Guo se rebelle contre ses maitres, et plus grave encore, s'enfuit et les trahit en prenant un autre maître. Il pèche de la sorte contre une hiérarchie «qui est voulue par le Ciel et la Terre» (ibid., chap. 11), et contre le devoir de loyauté, devoir résumé par la formule proverbiale, dûment rappelée dans le roman: «maître d'un jour, maître pour toujours» (ibid., chap. 11).

Comme si cela n'était pas suffisamment choquant, il tombe amoureux de la femme qui l'a pris comme disciple, la belle Fille-Dragon (Xiaolongnü), et se propose de l'épouser. Fille-Dragon a recueilli Yang Guo et lui a enseigné les arts martiaux, et même si elle n'est pas beaucoup plus âgée que le jeune homme (elle a vraisemblablement quatre ou cinq ans de plus que lui), il n'en demeure pas moins qu'elle est devenue son shifu (son «père-maître»), à l'issue d'un rituel en bonne et due forme (ibid., chap. 5). De ce fait, par rapport à Yang Guo, elle appartient à une génération précédente (qianbei): elle s'adresse d'ailleurs à lui en l'appelant «Guo'er», à la façon dont des parents appelleraient leur enfant, et non pas «jeune frère» ou «disciple-cadet» (shidi), comme le voulait l'usage pour des personnes de même génération. L'amour et le projet de mariage entre Yang Guo et Fille-Dragon va donc à l'encontre des règles de séparation entre les générations.

Il s'agit d'une question grave, qui fait l'objet d'une bonne partie du chapitre 14 , intitulé d'une manière révélatrice «Grande défense de la morale» (li jiao da fang). C'est Huang Rong, la gamine insolente de La Légende du héros chasseur d'aigles rencontrée ci-dessus, qui incarne ici les valeurs traditionnelles:

[Huang Rong] se rendit compte que Yang Guo et Fille-Dragon étaient très tendrement attachés l'un à l'autre, mais en même temps ils affichaient clairement une relation de maitre à disciple - était-il possible que leur comportement ait été déviant au point de violer à ce point les règles? C'était pour le moins difficile à croire; même si Yang Guo n'était pas un modèle de rectitude, il ne pouvait pas agir aussi inconsidérément. (ibid., chap. 14)

L'expression que nous avons traduite par «violer les règles » est nilun - mais on aurait presque pu traduire par «incestueux», tant la relation entre un maître et son disciple est choquante, puisqu'elle s'apparente à une relation parents/enfants. Huang Rong exprime avec force l'interdit que Yang Guo se propose de violer:

Très bien, puisque tu veux que je sois claire, je vais parler sans détour. Puisque FilleDragon est ton maître, elle est ton aînée, et tout sentiment de type homme-femme est impossible entre vous. (ibid.) 


\section{Yang Guo proteste:}

En quoi mon comportement vous gêne-t-il ? À qui ai-je fait du tort? [Fille-Dragon] m'a certes enseigné ses techniques de combat, mais je veux quand même l'épouser. Vous pouvez me couper en morceaux, je la veux quand même pour femme. (ibid.)

Cet argument de Yang Guo peut s'interpréter comme une intrusion de la modernité dans un monde traditionnel: il s'agit d'une affirmation d'individualisme face au poids des contraintes sociales, d'une attitude qui correspond à l'idée (occidentale moderne) selon laquelle notre liberté doit être garantie tant que, par notre comportement, on ne nuit pas à autrui. Après de nombreuses aventures et de longues séparations, Yang Guo et Fille-Dragon se retrouvent et se marient: Jin Yong a ici, d'une manière très peu conforme au confucianisme et aux valeurs «asiatiques », sacrifié la morale collective sur l'autel du bonheur personnel. Il s'agit d'une remise en cause d'un modèle qui privilégie la famille, le groupe, la société (et donc le père et les chefs), par rapport aux individus.

Le modèle patriarcal ressort quelque peu affaibli de romans comme Le Cerf et le Tripode ou Les Compagnons aux aigles merveilleux. Mais ce qui nuit sans doute le plus à la figure du père dans l'œuvre de Jin Yong, c'est tout simplement l'absence du géniteur: nous l'avons dit, bien des héros sont orphelins, ou affublés de pères indignes, c'est-à-dire de pères qui n'en sont pas. Bien souvent, on ne sait rien de leurs parents: c'est le cas de Yuan Chengzhi dans L'Épée tachée de sang royal (Bixue jian, 1956), de la jeune Li Wenxiu dans Vers l'Ouest sur un cheval blanc (Baima xiao xifeng, 1961), de Linghu Chong dans Aventurier fier et souriant, ou encore de «Petit Bâtard» dans La Marche des héros (Xiake xing, 1965) - Petit Bâtard n'a même pas de nom, et sa paternité demeure un mystère jusqu'au bout. Dans d'autres cas, les pères sont des parents convenables et aimables, mais qui se dépêchent de disparaître au début du récit: la mort du père de Guo Jing dans La Légende du héros chasseur d'aigles, celle des parents de Lin Pingzhi dans Aventurier fier et souriant, sont de ce point de vue typiques. Certes, les maîtres d'arts martiaux remplacent les pères, nous l'avons dit, mais si le maître possède l'autorité du père, il n'est pas une figure paternelle complète. Les maîtres d'arts martiaux disposent de l'autorité et des droits normalement réservés à un géniteur, mais ils n'ont pas les mêmes devoirs; et ils n'aiment pas forcément les disciples placés sous leur protection. Bien des maîtres se comportent de manière infâme à l'égard de leurs disciples, à l'image de l'un des plus célèbres d'entre eux, l'hypocrite Yue Buqun dans Aventurier fier et souriant, ou encore du maître de Petit Bâtard dans La Marche des héros, qui, pour se débarrasser du jeune garçon, lui enseigne volontairement des techniques funestes pour sa santé.

Lorsque les pères ne sont pas absents, ils ne sont guère plus dignes que les maîtres mentionnés ci-dessus. Duan Yu, l'un des héros de Demi-dieux et 
semi-dragons, a deux pères: l'homme qui l'a élevé est un personnage plutôt avenant, mais c'est en même temps un séducteur qui abandonne derrière lui une nuée de maîtresses qui ne songent plus qu'à le retrouver, le séquestrer ou s'en venger (ces mobiles représentent une bonne part de l'action du roman); quant au père biologique de Duan Yu, Duan Yanqing, que nous avons rencontré ci-dessus, il n'est autre que l'ennemi mortel de la famille, le principal des «Quatre grands méchants» du monde des montagnes et des lacs, un assassin sans scrupules qui ne songe qu'à retrouver son trône de Dali. Le père de Yang Guo est un traître et un meurtrier. Le père de Ren Yingying, l'héroïne de Aventurier fier et souriant, comme celui de Huang Rong dans La Légende du héros chasseur d'aigles sont à peine plus convenables: ils sont tous deux associés aux sectes hérétiques et réputés pour leur cruauté. Les actes de ces pères pèsent sur le récit et le destin des personnages, parfois durant des générations; plusieurs destins de femmes, notamment, sont brisés en raison du comportement de leur géniteur - c'est le cas de Yuan Ziyi, déjà rencontrée plus haut, dans La Biographie non officielle de Renard. Notons que, d'une façon générale, les filles et les mères paraissent se comporter d'une manière beaucoup plus morale que leurs comparses masculins, même si l'œuvre ne manque pas non plus de femmes criminelles, perverses ou folles.

Le statut du père chez Jin Yong est donc ambigu, entre présence et absence, ou prestige et indignité. Cette ambiguïté reflète sans doute une tension entre tradition et modernité, c'est-à-dire le problème posé par des valeurs patriarcales, qui restent des repères, mais qui ne sont pas sorties indemnes des critiques des intellectuels du Quatre Mai et de la confrontation avec l'individualisme occidental.

Le roman d'arts martiaux possède lui-même une dimension anachronique, avec un matériau (les intrigues, les époques, les personnages) qui paraît relever de la tradition, mais une tradition réinventée (comme toutes les traditions) par des auteurs modernes à l'intention de lecteurs modernes. Cette tension se retrouve même dans des romans d'arts martiaux très récents. Nous nous contenterons d'un exemple, celui de Lune brisée (Poyue), un roman signé de Ling Chen et datant de 2008 qui, en ce qui concerne les valeurs patriarcales, ne tranche guère avec des œuvres plus anciennes comme celles de Jin Yong. On y voit ainsi un grand-père qui veut imposer un mariage arrangé à son petit-fils. Lorsque des proches essaient de prendre la défense du jeune homme, qui n'aime pas la fille qui lui est promise, le grand-père s'emporte:

En quoi est-ce un problème? [Il] ne l'aime pas, il en épousera plus tard une autre qu'il aimera; ou bien il s'habituera peu à peu à elle et finira par l'aimer. En quoi est-ce un problème? [...] Ne sommes-nous pas tous passés par là? (Роуиe 98-99) 
Ce qui est frappant, c'est que Lune brisée se libère par d'autres aspects des contraintes du genre du roman d'arts martiaux: l'œuvre est influencée par le roman fantastique occidental, et l'histoire se déroule dans un monde imaginaire - beaucoup plus imaginaire que la Chine «historique» des romans de Jin Yong. Un dialogue comme celui qui précède est donc particulièrement révélateur, parce qu'il n'était pas nécessaire. On ne peut donc expliquer la permanence des valeurs patriarcales dans ce type d'œuvres uniquement en raison des contraintes liées à un genre particulier.

On retrouve des tensions analogues dans des genres populaires beaucoup plus susceptibles de remettre en cause la tradition. Afin, précisément, de montrer que les ambiguïtés identifiées dans l'œuvre de Jin Yong et dans d'autres romans d'arts martiaux ne s'expliquent pas seulement par des contraintes de genre, nous nous intéresserons, pour conclure cet article, à un autre type d'œuvre populaire, le roman sentimental (yanqing xiaoshuo), genre en première apparence beaucoup plus «moderne» que le roman d'arts martiaux.

Les romans sentimentaux mettent fréquemment en scène des conflits entre parents et enfants, parfois avec une dimension critique, voire revendicatrice. Le roman sentimental hongkongais passe pour particulièrement féministe; la femme y est souvent «forte et dure» (qiangying) ${ }^{24}$. Mais les représentations traditionnelles ne sont pas complètement dépassées. Ainsi, chez une Cen Kailun, les femmes sont fortes avant le mariage - mais un peu comme chez Jin Yong, elles tendent à se ranger une fois qu'elles sont mariées. Chez Yi Shu ou Liang Fengyi, les femmes obtiennent des diplômes et réussissent dans les sphères masculines, voire affichent un certain mépris vis-à-vis des hommes; elles n'hésitent pas à mener des vies non conformes aux valeurs traditionnelles. Le mariage, cependant, demeure important, voire incontournable, pour ces femmes. Et d'une façon plus générale, même pour les héroïnes les plus libérées, l'émancipation paraît plus facile à revendiquer qu'à vivre.

Ces auteures sont très prolifiques, et il n'est pas question ici de tenter une synthèse des idées mises en avant dans leurs romans. D'une façon très impressionniste, nous nous contenterons de quelques commentaires sur un roman de Yi Shu, Xibao, publié pour la première fois en 1986, et souvent présenté comme l'une des deux ou trois œuvres les plus représentatives de cette auteure ${ }^{25}$. Ce roman montre bien les ambiguïtés dans le traitement du père et du rapport au père dans ce type de littérature. Xibao, l'héroïne qui donne son nom au roman, est une jeune femme qui, à première vue, paraît très moderne. Elle a fait des études en Angleterre, c'est

24. Sur le «roman de femmes fortes» (nü qiangren xiaoshuo) hongkongais, voir Tang Zhesheng (2007) : 131-144, auquel nous empruntons les informations de ce paragraphe.

25. $C f$. (en chinois) la «liste partielle des principales auteures hongkongaises » www.hudong. com/wiki/\%E4\%BA\%A6\%E8\%88\%92 (consulté le 14 février 2011). 
«une femme on ne peut plus réaliste ${ }^{26} »$, une forte personnalité, qui professe des idées que l'on peut qualifier de «féministes»:

Un homme aura beaucoup de femmes dans sa vie. Une femme aura beaucoup d'hommes dans sa vie. Autrefois, les femmes pouvaient, assises dans la douceur de leur boudoir, broder leur vie durant; cette époque est révolue. (Xibao: 33)

L'affirmation de valeurs féministes est bien entendu une menace pour l'ordre patriarcal traditionnel; l'homme en général, et le père en particulier, perd le monopole du pouvoir. Les femmes ne servent plus les hommes, elles s'en servent, comme le dit non sans cynisme Xibao:

Toute femme a dans sa vie besoin d'hommes lui servant de marchepied. (ibid.: 21)

Le roman abonde en jugements du même acabit, généralement dans la bouche de Xibao, qui de ce point de vue tranche radicalement avec la femme «traditionnelle». Mais ce cynisme cache une détresse, ou du moins révèle un malaise: Xibao n'assume qu'en partie sa condition de femme libérée. Elle continue à se voir à travers le regard des hommes, en l'occurrence comme une marchandise :

Peu m'importe de vendre ma jeunesse, si je ne la vends pas elle passera quand même. (ibid.: 49)

Lorsqu'on lui dit que rien ne la force à rester avec un homme qu'elle n'aime pas, elle répond:

Comment ça? Ma situation financière me force à rester avec lui. N'est-ce pas une raison suffisante?» (ibid.: 21)

Cette opinion réaffirme, peut-être sous une forme ironique, un rapport traditionnel entre hommes et femmes. Xibao ne remet d'ailleurs pas en cause l'importance du mariage pour les femmes:

Toutes les femmes devraient se marier et trouver un moyen pour que leur mari gagne de l'argent pour les entretenir. (ibid.: 9)

Elle-même se donne clairement pour but le mariage:

Rencontrer ${ }^{27}$ des hommes, c'est vraiment facile, et je n'ai pas de souci à me faire sur ce plan durant les vingt-cinq prochaines années. Ce qui est difficile, c'est de les pousser au mariage. Dans tous les cas, la plus grande marque de respect d'un homme envers une femme, c'est de la demander en mariage [...]. (ibid.: 98)

26. $C f$. pour ce jugement www.hudong.com/wiki/\%E4\%BA\%A6\%E8\%88\%92 (consulté le 14 février 2011).

27. «Rencontrer», peng, possède ici une connotation sexuelle. 
Dans le mariage, l'amour n'est pas forcément indispensable, parce que «l'amour, c'est du luxe» (ibid.: 63). Sous des dehors provocants, le roman reproduit donc une vision très conventionnelle du mariage, avec une répartition traditionnelle des rôles, les hommes assurant la sécurité matérielle et les femmes se cantonnant pour leur part dans un rôle passif. Notons d'ailleurs que le prénom de l'héroïne, Xibao (littéralement «trésor heureux » ou «bonheur précieux »), peut lui-même se comprendre comme une allusion au mariage - le caractère $x i$, «heureux», «joie», apparaît dans de multiples expressions liées au mariage.

En fin de compte, Xibao accepte de devenir la maîtresse d'un homme très riche qui l'entretient. Elle le fait notamment pour des raisons économiques, et sa posture est celle d'une concubine:

Voilà pourquoi je suis la meilleure des maîtresses. Quand il a du temps, je lui tiens compagnie. Et quand il n'a pas de temps, je l'attends. (ibid.: 260)

Une autre ambiguïté, sans doute plus profonde encore, de cette femme élevée comme une femme moderne, mais qui endosse des valeurs traditionnelles, est son rapport au père. Son père biologique, «le plus actif des célibataires du district de Central», «le plus grand menteur au monde» (ibid.: 223), s'est contenté « de l'engendrer, mais sans l'élever» (ibid. : 208). Il ne s'est jamais occupé d'elle, et ne lui rappelle leur relation père-fille que pour lui extorquer de l'argent $(C f$. ibid. : 211), dans une relation inversée où c'est la fille (avec l'argent de son amant) qui soutient son géniteur. De fait, Xibao ne se reconnaît aucun père:

Ma mère a vraiment eu une vie difficile, elle a épousé trop tôt mon père, ce fêtard qui gaspillait toute son énergie dans une vie dissolue, un parvenu qui après avoir emprunté de l'argent allait le dépenser au Ritz en danses; lorsque le Ritz s'est transformé en résidence de luxe il a divorcé d'avec ma mère, à l'époque je savais à peine marcher... Je ne l'ai jamais vraiment vu, et je ne regrette rien - mon nom de famille est Jiang, c'est le nom de famille de ma mère. Le nom de mon père n'a aucune importance pour moi. (ibid.: 9)

À première vue, le père est donc absent - mais il est tout de même présent puisqu'en définitive, c'est par la faute de son géniteur que Xibao est malheureuse. Et ce père raté doit d'une certaine manière être remplacé. Chez Jin Yong, les pères absents étaient remplacés par les maîtres d'arts martiaux. Xibao se trouve pour sa part un amant riche, qui a quarante ans de plus qu'elle - alors qu'elle-même méprise son propre père «qui couche avec une secrétaire qui a l'âge de sa propre fille». Cet amant âgé, bien entendu, remplace le père absent ; lui-même reconnaît qu'il aurait aimé l'avoir comme fille :

Tu es décidée, posée, résolue, c'est toi ma véritable fille. (ibid.: 185) 
À quoi elle répond:

Et toi tu me traites bien, tout à fait aussi bien qu'un père traite sa fille, tu es vraiment bien pour moi. (ibid.)

Le jeune Allemand qui, à un moment du récit, courtise Xibao lui demande d'ailleurs ironiquement si son «père» (en parlant de l'amant de la jeune fille) accepterait qu'il fréquente sa «fille» (ibid.: 188-189). En réalité, Xibao n'a ni père ni mari, alors que, selon ses propres dires, «ce sur quoi une femme peut se reposer toute sa vie, c'est seulement son père et son mari» (ibid.: 122). Son amant âgé joue les deux rôles, dans les deux cas d'une manière incomplète.

Xibao est donc instructif de l'ambiguïté du rapport au père, dans une ville «chinoise» moderne, et c'est une ambiguïté que l'on retrouve fréquemment dans la littérature populaire contemporaine. Cette littérature reflète en effet, on l'aura compris, la société et ses évolutions de toutes sortes de manières; et si par certains aspects le genre tend au conservatisme, par d'autres il peut se montrer très progressiste, voire subversif. On trouve les deux facettes chez Jin Yong et chez Yi Shu. Et la littérature très récente, celle des années 2000, est de ce point de vue révélatrice, dans un contexte politique de réaffirmation de valeurs patriarcales.

En effet, depuis quelques années, en république populaire de Chine, l'État tente de promouvoir des valeurs «traditionnelles» fréquemment associées au confucianisme. Un bon exemple est la piété filiale, que le gouvernement envisage d'inscrire dans la $l^{2} i^{28}$. Le premier but d'une telle disposition est sans doute d'inviter les enfants à s'occuper de leurs parents, dans une société de plus en plus individualiste ${ }^{29}$. Mais la promotion de la piété filiale s'inscrit dans un programme plus large de mesures servant les intérêts du pouvoir: la piété filiale est à certains égards positive, mais en encourageant à l'obéissance, elle peut aussi servir les intérêts d'un pouvoir autoritaire - Confucius lui-même avait déjà compris que le respect des parents est le fondement de la soumission au pouvoir politique ${ }^{30}$.

Dans ce contexte, la littérature populaire révèle fort bien les tensions entre l'idéologie officielle et la société réelle, notamment dans les villes, où les manières de vivre et les formes d'organisation familiale sont de moins en moins «confucianistes»-que ce soit pour le meilleur ou le pire. Certains romans peuvent même s'interpréter contre une forme de résistance contre cette

28. Cf. China Daily, 11 janvier 2011 : http://europe.chinadaily.com.cn/opinion/2011-01/11/ content_11824063.htm (consulté le 14 février 2011).

29. Sur le recul de la piété filiale dans la société chinoise, voir les résultats d'une étude récente publiée dans le China Daily, 13 janvier 2011, http://europe.chinadaily.com.cn/ opinion/2011-01/13/content_11856185.htm (consulté le 14 février 2011).

30. Cf. Entretiens de Confucius, chap. 2.2. 
réaffirmation de valeurs patriarcales. À titre d'exemple, mentionnons la vogue, depuis le milieu des années 2000, des «romans de pilleurs de tombes» (daomu xiaoshuo), genre hybride entre le roman d'arts martiaux, le roman fantastique et le roman de fantômes, qui dépeint de jeunes héros bravant tous les dangers pour s'approprier les trésors cachés dans les sépultures dont regorge le soussol chinois. Le pillage de tombes est un sacrilège particulièrement choquant dans un pays comme la Chine, où le culte des ancêtres (c'est-à-dire le culte des pères) a joué un rôle si important. Au risque de sombrer dans la psychologie à bon marché, on interprétera peut-être le succès de ce genre littéraire auprès d'un public adolescent comme une forme d'iconoclasme libérateur vis-à-vis du passé, des hiérarchies et, par prolongement, de l'autorité des parents, dans une société de tiger moms qui mettent beaucoup de pression sur les enfants ${ }^{31}$.

L'humour contribue aussi dans certains romans à éroder la déférence à l'égard des valeurs patriarcales que l'idéologie officielle tente de remettre à l'honneur. Un bon exemple est Pas sérieux s'abstenir (Fei cheng wu rao, 2008), du metteur en scène Feng Xiaogang, tiré du film à succès du même nom ${ }^{32}$. Le héros du roman, Qin Fen, un quadragénaire célibataire qui rentre des USA, cherche une épouse par petites annonces. Une jeune femme répond en l'alléchant avec la description de son physique avantageux ; le rendez-vous est fixé dans un temple. Lors de leur rencontre, elle lui dit qu'elle a voulu le rencontrer dans un endroit aussi sacré pour que, à son retour au pays, il puisse «honorer ses ancêtres»; elle lui parle de piété filiale, demande où il a enterré son père et où il enterrera sa mère, parce qu'elle admire «les gens qui traitent filialement leurs parents»: un homme doit avoir un «cœur filial» (xiaoxin) et trouver le meilleur endroit pour enterrer ses parents. Un beau discours, auquel Qin Fen est sensible: la jeune femme lui vend une concession funéraire pour 300000 yuan - pour cette jeune courtière en immobilier funéraire, la piété filiale est devenue un simple argument de marketing.

31. La sévérité (supposée) de l'éducation à la chinoise est devenue récemment un enjeu de politique intérieure aux États-Unis, suite à la publication par la sino-américaine Amy Chua de Battle Hymn of the Tiger Mother, ouvrage dans lequel elle oppose ses méthodes impitoyables d'éducation, qu'elle présente comme typiquement chinoises, à la permissivité des parents américains. Amy Chua a fait la une du magazine Time du 31 janvier 2011.

32. Ce film fut l'un des plus grands succès commerciaux du cinéma chinois, avec 300 millions d'entrées après trois semaines d'exploitation. 


\section{Biblographie}

Pour les éditions et la traduction des titres des romans de Jin Yong, $c f$. supra, note 12.

Altenburger Roland (2009). The Sword or the Needle: The Female Knight-errant (xia) in Traditional Chinese Narrative. Bern: Peter Lang.

Artiaga Loïc (dir.) (2008). Le Roman populaire, 1836-1960. Des premiers feuilletons aux adaptations télévisuelles. Paris: Autrement.

(Ba Jin 巴金) Pa Kin (1979). Famille. Paris: Flammarion.

Barthlein Thomas (1999). «Mirrors of Transition»: Conflicting Images of Society in Change from Popular Chinese Social Novels, 1908-1930. Modern China, $\mathrm{n}^{\mathrm{o}} 25$ (2): 204-228.

Chua Amy (2011). Battle Hymn of the Tiger Mother. New York: Penguin.

FENG Xiaogang 冯小刚 (2008). Fei cheng wu rao 非诚勿扰 [Pas sérieux s'abstenir]. Wuhan: Changjiang wenyi chubanshe.

Gu Long 古龍 (1991). Duoqing jianke wuqing jian 多情劍客無情劍 [Le héros est sensible mais son épée est insensible, 1968]. Shenzhen: Haitian chubanshe.

JiN Yong 金庸 (1955). Shu jian enchou lu 書劍恩仇錄 (Le Livre et l’Épée).

JIN Yong 金庸 (1957). Shediao yingxiong zhuan 射鵰英雄傳 (Le Héros chasseur d'aigles). Trad. Wang Jiann-Yuh (2004). La Légende du héros chasseur d'aigles. Paris: Éditions You-Feng (2 vol.).

JIN Yong 金庸 (1959). Shendiao xialï 神鵰俠侶 (Les Compagnons aux aigles merveilleux). JIN Yong 金庸 (1960). Fei Hu waizhuan 飛狐外傳 (La Biographie non officielle de Renard).

JIN Yong 金庸 (1961). Yitian tulong ji 倚天屠龍記 (Le Céleste Tueur de dragons).

JIN Yong 金庸 (1963). Tianlong babu 天龍八部 (Demi-dieux et semi-dragons).

JIN Yong 金庸 (1965). Xiake xing 俠客行 (La Marche des héros).

JIN Yong 金庸 (1967). Xiao'ao jianghu 笑傲江湖 (Aventurier fier et souriant).

JIN Yong 金庸 (1969-1972). Lu ding ji 鹿鼎記 (Le Cerf et le Tripode). Trad. John MinfoRD et Rachel May, Cha, Louis (1997-2003). The Deer and the Cauldron. Oxford: Oxford University Press (3 vol.).

LING Chen 淩晨 (2008). Роуиe 破月 [Lune brisée]. Changchun: Shidai wenyi chubanshe (2 vol.).

PIckowicz Paul C. (1991). The Theme of Spiritual Pollution in Chinese Films of the 1930s. Modern China, $\mathrm{n}^{\circ} 17$ (1): 38-75.

TANG Zhesheng 汤哲声 (dir.) (2007). Zhongguo dangdai tongsu xiaoshuo shilun 中国当 代通俗小说史论 [Histoire raisonnée du roman populaire chinoise contemporain]. Beijing: Beijing daxue chubanshe.

TANG Zhesheng 汤哲声 (2008). Zhongguo xiandai tongsu xiaoshuo sibianlu 中国现代 通俗小说思辨录 [Mémoire sur le roman populaire chinois moderne]. Beijing: Beijing daxue chubanshe.

YI Shu 亦舒 (2007). Xibao 喜宝. Beijing: Xin shijie chubanshe.

Zhang Yinde (2003). Jin Yong et la Chevalerie sans frontières. In ZHANG Yinde, Le Monde romanesque chinois au XXe siècle. Paris: Honoré Champion : 317-335.

Zhou Shoujuan 周瘦鹃 (2011). Fu zi 父子 [Père et fils]. In Zhou Shoujuan wenji 周瘦鹃 文集 [Euvres de Zhou Shoujuan]. Shanghai: Wenhui chubanshe, vol.I : 319-325. 
Nicolas Zufferey

\section{Glossaire}

Ba Jin 巴金

Baimaxiao xifeng 白馬嘯西風

bao 報

Bixue jian 碧血劍

Cen Kailun 岑凱倫

chun wenxue 純文學

Dali 大理

daomu xiaoshuo 盜墓小說

Duan Yanqing 段延慶

Duan $\mathrm{Yu}$ 段譽

Fei cheng wu rao 非誠勿擾

Fei Hu wai zhuan 飛狐外傳

Feng Tiannan 鳳天南

Feng Xiaogang 馮小剛

$F u z i$ 父子

Guo Jing 郭靖

Guo Moruo 郭沫若

Guo'er 過兒

Haishang hua liezhuan 海上花列傳

Huang Rong 黃蓉

Huang l'Herboriste 黃藥師

$\mathrm{Hu}$ Fei 胡斐

Jin Yong 金庸

Liang Fengyi 梁鳳儀

li jiao da fang 禮教大防

Li Ji, «Nei ze» 禮記 內則

Liji «Tangong, shang» 禮記檀弓上

Ling Chen 凌晨

Linghu Chong 令狐沖

Li Wenxiu 李文秀

Lin Pingzhi 林平之

Lu ding ji 鹿鼎記

Lu Xun 魯迅

Mao Dun 茅盾

Mei Chaofeng 梅超風

nilun 逆倫

Poyue 破月

Qianbei 前輩

qiangdao 強盜

qiangying 強硬

Qin Fen 秦奮

Ren Woxing 任我行 
Ren Yingying 任盈盈

Shediao yingxiong zhuan 射雕英雄傳

Shendiao xialü 神雕俠侶

shidi 師弟

Shifu 師父

Shun 舜

Tian Boguang 田伯光

Tianlong babu 天龍八部

tongsu wenxue 通俗文學

Wang Dungen 王鈍根

Wang Yuyan 王語嫣

Wei Xiaobao 韋小寶

wuxia xiaoshuo 武俠小說

Xiake xing 俠客行

xiao 孝

Xiao'ao jianghu 笑傲江湖

Xiaolongnü 小龍女

xiaoxin 孝心

Xibao 喜寶

xin wuxia xiaoshuo 新武俠小說

Xixia 西夏

Yang Guo 楊過

yanqing xiaoshuo 言情小說

Yan Yuan 顏淵

Yi Shu 亦舒

Yuan Chengzhi 袁承志

yuanyang hudie pai 鴛鴍蝴蝶派

Yuan Ziyi 袁紫衣

Yue Buqun 岳不群

Zhang Yinde 張殷德

Zheng Zhenduo 鄭振鐸

Zhou Shoujuan 周瘦鵑 
